\title{
Simple interpretations of lepton anomalies in the lepton-specific inert two-Higgs-doublet model
}

\author{
Xiao-Fang Han, ${ }^{1}$ Tianjun Li, ${ }^{2,3}$ Lei Wang, ${ }^{1}$ and Yang Zhang ${ }^{4}$ \\ ${ }^{1}$ Department of Physics, Yantai University, Yantai 264005, People's Republic of China \\ ${ }^{2}$ CAS Key Laboratory of Theoretical Physics, Institute of Theoretical Physics, \\ Chinese Academy of Sciences, Beijing 100190, People's Republic of China \\ ${ }^{3}$ School of Physical Sciences, University of Chinese Academy of Sciences, \\ Beijing 100049, People's Republic of China \\ ${ }^{4}$ ARC Centre of Excellence for Particle Physics at the Tera-scale, School of Physics and Astronomy, \\ Monash University, Melbourne, Victoria 3800, Australia
}

(Received 11 December 2018; published 24 May 2019)

\begin{abstract}
There exist about $3.7 \sigma$ positive and $2.4 \sigma$ negative deviations in the muon and electron anomalous magnetic moments $(g-2)$. Also, some ratios for lepton universality in $\tau$ decays have almost $2 \sigma$ deviations from the Standard Model. In this paper, we propose a lepton-specific inert two-Higgs-doublet model. After imposing all the relevant theoretical and experimental constraints, we show that these lepton anomalies can be explained simultaneously in many parameter spaces with $m_{H}>200 \mathrm{GeV}$ and $m_{A}\left(m_{H^{ \pm}}\right)>500 \mathrm{GeV}$ for appropriate Yukawa couplings between leptons and inert Higgs. The key point is that these Yukawa couplings for $\mu$ and $\tau / e$ have opposite sign.
\end{abstract}

DOI: $10.1103 /$ PhysRevD.99.095034

\section{INTRODUCTION}

The Standard Model (SM) describes the elementary particles, as well as the fundamental interactions between them. In particular, such description is sensitive to the quantum corrections. For example, since Schwinger's seminar calculation of the electron anomalous magnetic moment $a_{e}=\alpha / 2 \pi$ [1], the charged lepton anomalous magnetic moments have become the powerful precision tests of quantum electrodynamics (QED), and subsequently the full SM. The muon anomalous magnetic moment $g-2$ has been a long-standing puzzle since the announcement by the E821 experiment in 2001 [2]. The experimental value has an approximate $3.7 \sigma$ discrepancy from the SM prediction [3]

$$
\Delta a_{\mu}=a_{\mu}^{\exp }-a_{\mu}^{\mathrm{SM}}=(274 \pm 73) \times 10^{-11} .
$$

Very recently, an improvement in the measured mass of atomic cesium used in conjunction with other known mass ratios and the Rydberg constant leads to the most precise value of the fine structure constant [4]. As a result, the experimental value of the electron $g-2$ has a $2.4 \sigma$ deviation from the SM prediction [5-7]

Published by the American Physical Society under the terms of the Creative Commons Attribution 4.0 International license. Further distribution of this work must maintain attribution to the author(s) and the published article's title, journal citation, and DOI. Funded by SCOAP ${ }^{3}$.

$$
\Delta a_{e}=a_{e}^{\mathrm{exp}}-a_{e}^{\mathrm{SM}}=(-87 \pm 36) \times 10^{-14},
$$

which is opposite in sign from the muon $g-2$.

The lepton flavor universality (LFU) in the $\tau$ decays is an excellent way to probe new physics. The HFAG collaboration reported three ratios from pure leptonic processes, and two ratios from semihadronic processes, $\tau \rightarrow \pi / K \nu$ and $\pi / K \rightarrow \mu \nu[8]$ :

$$
\begin{array}{rlrl}
\left(\frac{g_{\tau}}{g_{\mu}}\right) & =1.0011 \pm 0.0015, & \left(\frac{g_{\tau}}{g_{e}}\right)=1.0029 \pm 0.0015 \\
\left(\frac{g_{\mu}}{g_{e}}\right) & =1.0018 \pm 0.0014, \quad\left(\frac{g_{\tau}}{g_{\mu}}\right)_{\pi}=0.9963 \pm 0.0027 \\
\left(\frac{g_{\tau}}{g_{\mu}}\right)_{K} & =0.9858 \pm 0.0071,
\end{array}
$$

where the ratios of $g_{\tau} / g_{e}$ and $\left(\frac{g_{\tau}}{g_{\mu}}\right)_{K}$ have almost $2 \sigma$ deviations from the SM.

Muon $g-2$ anomaly can be simply explained in the lepton-specific two-Higgs-doublet model (2HDM) and aligned 2HDM. However, the tree-level diagram mediated by the charged Higgs gives a negative contribution to the decay $\tau \rightarrow \mu \nu \bar{\nu}$ [9-11], which will raise the discrepancy in the LFU in $\tau$ decays. In addition, these two types of 2HDM do not explain the muon and electron $g-2$ simultaneously since there is an opposite sign between them. Therefore, we shall propose a lepton-specific inert $2 \mathrm{HDM}$ to explain all three anomalies of muon and electron $g-2$ as well as 
LFU in $\tau$ decay simultaneously. In our model, for the extra Higgses $\left(H, A, H^{ \pm}\right)$, the Yukawa couplings for $\mu$ and $\tau / e$ have opposite sign. In 2012, Giudice et al. used the approach of the effective operator to discuss the contributions of light scalar to the muon and electron $g-2$. The contributions of two-loop Barr-Zee-type diagrams can be positive or negative depending on the relative sign of the Yukawa couplings for muon, electron, and tau [12]. Although the muon and electron $g-2$ have been addressed simultaneously in a few recent papers [13-17], it seems to us that our model is simpler from the renormalized theory point of view.

\section{LEPTON-SPECIFIC INERT 2HDM}

We introduce an inert Higgs doublet $\Phi_{2}$ in the SM as well as a discrete $Z_{2}$ symmetry under which $\Phi_{2}$ is odd while all the SM particles are even. The scalar potential for the SM Higgs field $\Phi_{1}$ and inert doublet $\Phi_{2}$ is

$$
\begin{aligned}
\mathrm{V}= & Y_{1}\left(\Phi_{1}^{\dagger} \Phi_{1}\right)+Y_{2}\left(\Phi_{2}^{\dagger} \Phi_{2}\right)+\frac{\lambda_{1}}{2}\left(\Phi_{1}^{\dagger} \Phi_{1}\right)^{2}+\frac{\lambda_{2}}{2}\left(\Phi_{2}^{\dagger} \Phi_{2}\right)^{2} \\
& +\lambda_{3}\left(\Phi_{1}^{\dagger} \Phi_{1}\right)\left(\Phi_{2}^{\dagger} \Phi_{2}\right)+\lambda_{4}\left(\Phi_{1}^{\dagger} \Phi_{2}\right)\left(\Phi_{2}^{\dagger} \Phi_{1}\right) \\
& +\left[\frac{\lambda_{5}}{2}\left(\Phi_{1}^{\dagger} \Phi_{2}\right)^{2}+\text { H.c. }\right] .
\end{aligned}
$$

We focus on the $C P$-conserving case where all $\lambda_{i}$ are real. The two complex scalar doublets can be written as

$$
\Phi_{1}=\left(\begin{array}{c}
G^{+} \\
\frac{1}{\sqrt{2}}\left(v+h+i G_{0}\right)
\end{array}\right), \quad \Phi_{2}=\left(\begin{array}{c}
H^{+} \\
\frac{1}{\sqrt{2}}(H+i A)
\end{array}\right) .
$$

The $\Phi_{1}$ field has the vacuum expectation value (VEV) $v=246 \mathrm{GeV}$, and the $\mathrm{VEV}$ of $\Phi_{2}$ field is zero. $Y_{1}$ is fixed by the scalar potential minimization condition. The $H^{+}$and $A$ are the mass eigenstates of the charged Higgs boson and $\mathrm{CP}$-odd Higgs boson. Their masses are given as

$m_{H^{ \pm}}^{2}=Y_{2}+\frac{\lambda_{3}}{2} v^{2}, \quad m_{A}^{2}=m_{H^{ \pm}}^{2}+\frac{1}{2}\left(\lambda_{4}-\lambda_{5}\right) v^{2}$.

The $h$ and $H$ have no mixing, and they are two mass eigenstates of the $C P$-even Higgses. In this paper, we take the light $C P$-even Higgs $h$ as the SM-like Higgs. Their masses are given as

$m_{h}^{2}=\lambda_{1} v^{2} \equiv(125 \mathrm{GeV})^{2}, \quad m_{H}^{2}=m_{A}^{2}+\lambda_{5} v^{2}$.

The fermions obtain the mass terms from the Yukawa interactions with $\Phi_{1}$ :

$-\mathcal{L}=y_{u} \bar{Q}_{L} \tilde{\Phi}_{1} u_{R}+y_{d} \bar{Q}_{L} \Phi_{1} d_{R}+y_{l} \bar{L}_{L} \Phi_{1} e_{R}+$ H.c., where $Q_{L}^{T}=\left(u_{L}, d_{L}\right), L_{L}^{T}=\left(\nu_{L}, l_{L}\right), \tilde{\Phi}_{1}=i \tau_{2} \Phi_{1}^{*}$, and $y_{u}$, $y_{d}$ and $y_{\ell}$ are $3 \times 3$ matrices in family space. In addition, only in the lepton sector we introduce the $Z_{2}$ symmetrybreaking Yukawa interactions of $\Phi_{2}$,

$$
\begin{aligned}
-\mathcal{L}= & \sqrt{2} \kappa_{e} \bar{L}_{1 L} \Phi_{2} e_{R}+\sqrt{2} \kappa_{\mu} \bar{L}_{2 L} \Phi_{2} \mu_{R} \\
& +\sqrt{2} \kappa_{\tau} \bar{L}_{3 L} \Phi_{2} \tau_{R}+\text { H.c. }
\end{aligned}
$$

Such the $Z_{2}$ symmetry-breaking effect only for the lepton sector can be realized in the high-dimensional brane world scenario, which will be studied elsewhere. From Eq. (9), we can obtain the lepton Yukawa couplings of extra Higgses $\left(H, A\right.$, and $\left.H^{ \pm}\right)$. The neutral Higgses $A$ and $H$ have no couplings to $Z Z, W W$.

\section{NUMERICAL RESULTS}

According to Eqs. (6) and (7), the values of $\lambda_{1}, \lambda_{5}$ and $\lambda_{4}$ can be determined by $m_{h}(=125 \mathrm{GeV}), m_{H}, m_{A}$ and $m_{H^{ \pm}}$. $\lambda_{2}$ controls the quartic couplings of extra Higgses, but does not affect the physics observables. So we simply take $\lambda_{2}=\lambda_{1}$. Because the precision electroweak data favor small mass splitting between $m_{A}$ and $m_{H^{ \pm}}$, we simply choose $m_{A}=m_{H^{ \pm}}$. We employ the 2HDMC [18] to implement the theoretical constraints from vacuum stability, unitarity and perturbativity, as well as the constraints of the oblique parameters $(S, T, U)$. We scan over several key parameters in the following ranges

$$
0.5<\kappa_{\tau}<1, \quad-0.25<\kappa_{\mu}<0, \quad 0<\kappa_{e}<0.01,
$$

$200 \mathrm{GeV}<m_{H}<350 \mathrm{GeV}$,

$500 \mathrm{GeV}<m_{A}=m_{H^{ \pm}}<700 \mathrm{GeV}$.

In such ranges of $\kappa_{\tau}, \kappa_{\mu}$ and $\kappa_{e}$, the corresponding Yukawa couplings do not become nonperturbative. At the tree-level, the SM-like Higgs has the same couplings to the SM particles as the SM, and no exotic decay mode. The masses of extra Higgses are beyond the exclusion range of the searches for the neutral and charged Higgs at the LEP. Since the extra Higgses have no couplings to quarks due to $Z_{2}$ symmetry, we can safely neglect the limits from the observables of meson. The extra Higgs bosons are dominantly produced at the LHC via electroweak processes. We generate the Monte Carlo events using MG5_AMC-2.4.3 [19] with PYTHIA6 [20], and adopt the constraints from all the analysis for the $13 \mathrm{TeV}$ LHC in CHECKMATE 2.0.7 [21]. The latest multilepton searches for electroweakino [22-25] are further applied because of the dominated multilepton final states in our model.

In the model, the extra one-loop contributions to muon $g-2$ are given as [26]

$$
\Delta a_{\mu}(1 \text { loop })=\frac{1}{2 \pi^{2}} \sum_{i} \kappa_{\mu}^{2} r_{\mu}^{i} F_{i}\left(r_{\mu}^{i}\right)
$$



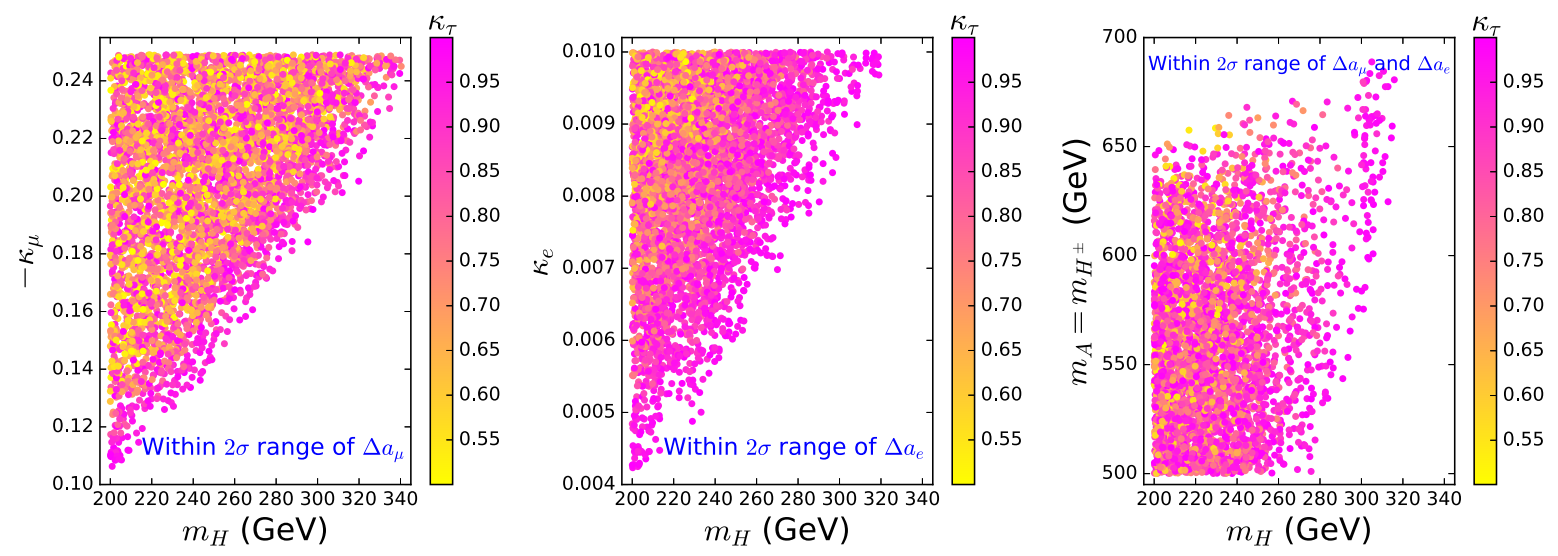

FIG. 1. The samples within $2 \sigma$ ranges of $\Delta a_{\mu}$ (left panel), $\Delta a_{e}$ (middle panel), and both $\Delta a_{\mu}$ and $\Delta a_{e}$ (right panel). All the samples satisfy the constraints of the theory and oblique parameters.

where $i=H, A, H^{ \pm}, r_{\mu}^{i}=m_{\mu}^{2} / M_{i}^{2}$. For $r_{\mu}^{i} \ll 1$ we have

$$
F_{H}(r) \simeq-\ln r-7 / 6, \quad F_{A}(r) \simeq \ln r+11 / 6,
$$

$F_{H^{ \pm}}(r) \simeq-1 / 6$.

The contributions of the two-loop diagrams with a closed fermion loop are given by

$$
\Delta a_{\mu}(2 \text { loop })=\frac{m_{\mu}}{8 \pi^{2}} \frac{\alpha_{\mathrm{em}}}{\pi} \sum_{i, \ell} Q_{\ell}^{2} \kappa_{\mu} \frac{\kappa_{\ell}}{m_{\ell}} r_{\ell}^{i} G_{i}\left(r_{\ell}^{i}\right),
$$

where $i=H, A, \ell=\tau$, and $m_{\ell}$ and $Q_{\ell}$ are the mass and electric charge of the lepton $\ell$ in the loop. The functions $G_{i}(r)$ are given in Refs. [27,28],

$$
\begin{aligned}
G_{H}(r) & =\int_{0}^{1} d x \frac{2 x(1-x)-1}{x(1-x)-r} \ln \frac{x(1-x)}{r}, \\
G_{A}(r) & =\int_{0}^{1} d x \frac{1}{x(1-x)-r} \ln \frac{x(1-x)}{r} .
\end{aligned}
$$

We also consider the contributions of the two-loop diagrams with a closed charged Higgs loop, and find that their contributions are much smaller than the fermion loop. The calculations of $\Delta a_{e}$ are similar to $\Delta a_{\mu}$, but for the contributions of the two-loop diagrams, we include both $\mu$ loop and $\tau$ loop.

The HFAG collaboration reported three ratios from pure leptonic processes, and two ratios from semihadronic processes, $\tau \rightarrow \pi / K \nu$ and $\pi / K \rightarrow \mu \nu$ [8]. In the model, we have the ratios

$$
\begin{aligned}
& \left(\frac{g_{\tau}}{g_{\mu}}\right)^{2} \equiv \bar{\Gamma}(\tau \rightarrow e \nu \bar{\nu}) / \bar{\Gamma}(\mu \rightarrow e \nu \bar{\nu}) \approx \frac{1+2 \delta_{\text {loop }}^{\tau}}{1+2 \delta_{\text {loop }}^{\mu}} \\
& \left(\frac{g_{\tau}}{g_{e}}\right)^{2} \equiv \bar{\Gamma}(\tau \rightarrow \mu \nu \bar{\nu}) / \bar{\Gamma}(\mu \rightarrow e \nu \bar{\nu}) \approx \frac{1+2 \delta_{\text {tree }}+2 \delta_{\text {loop }}^{\tau}}{1+2 \delta_{\text {loop }}^{\mu}} \\
& \left(\frac{g_{\mu}}{g_{e}}\right)^{2} \equiv \bar{\Gamma}(\tau \rightarrow \mu \nu \bar{\nu}) / \bar{\Gamma}(\tau \rightarrow e \nu \bar{\nu}) \approx 1+2 \delta_{\text {tree }} \\
& \left(\frac{g_{\tau}}{g_{\mu}}\right)_{\pi}^{2}=\left(\frac{g_{\tau}}{g_{\mu}}\right)_{K}^{2}=\left(\frac{g_{\tau}}{g_{\mu}}\right)^{2}
\end{aligned}
$$

where $\bar{\Gamma}$ denotes the partial width normalized to its SM value. $\delta_{\text {tree }}$ and $\delta_{\text {loop }}^{\tau, \mu}$ obtain corrections from the tree-level and one-loop diagrams mediated by the charged Higgs, respectively. They are given as $[9,11]$

$$
\begin{aligned}
\delta_{\text {tree }} & =\frac{v^{4} \kappa_{\tau}^{2} \kappa_{\mu}^{2}}{8 m_{H^{ \pm}}^{4}}-\frac{v^{2} m_{\mu}}{m_{H^{ \pm}}^{2} m_{\tau}} \kappa_{\tau} \kappa_{\mu} \frac{g\left(m_{\mu}^{2} / m_{\tau}^{2}\right)}{f\left(m_{\mu}^{2} / m_{\tau}^{2}\right)}, \\
\delta_{\text {loop }}^{\tau, \mu} & =\frac{1}{16 \pi^{2}} \kappa_{\tau, \mu}^{2}\left[1+\frac{1}{4}\left(H\left(x_{A}\right)+H\left(x_{H}\right)\right)\right],
\end{aligned}
$$

where $f(x) \equiv 1-8 x+8 x^{3}-x^{4}-12 x^{2} \ln (x), \quad g(x) \equiv 1+9 x-$ $9 x^{2}-x^{3}+6 x(1+x) \ln (x)$, and $H\left(x_{\phi}\right) \equiv \ln \left(x_{\phi}\right)\left(1+x_{\phi}\right) /$ $\left(1-x_{\phi}\right)$ with $x_{\phi}=m_{\phi}^{2} / m_{H^{ \pm}}^{2}$.

The correlation matrix for the above five observables is

$$
\left(\begin{array}{ccccc}
1 & +0.53 & -0.49 & +0.24 & +0.12 \\
+0.53 & 1 & +0.48 & +0.26 & +0.10 \\
-0.49 & +0.48 & 1 & +0.02 & -0.02 \\
+0.24 & +0.26 & +0.02 & 1 & +0.05 \\
+0.12 & +0.10 & -0.02 & +0.05 & 1
\end{array}\right)
$$

We perform $\chi_{\tau}^{2}$ calculations for these five observables. The covariance matrix constructed from the data of Eqs. (3) and (19) has a vanishing eigenvalue, and the corresponding 


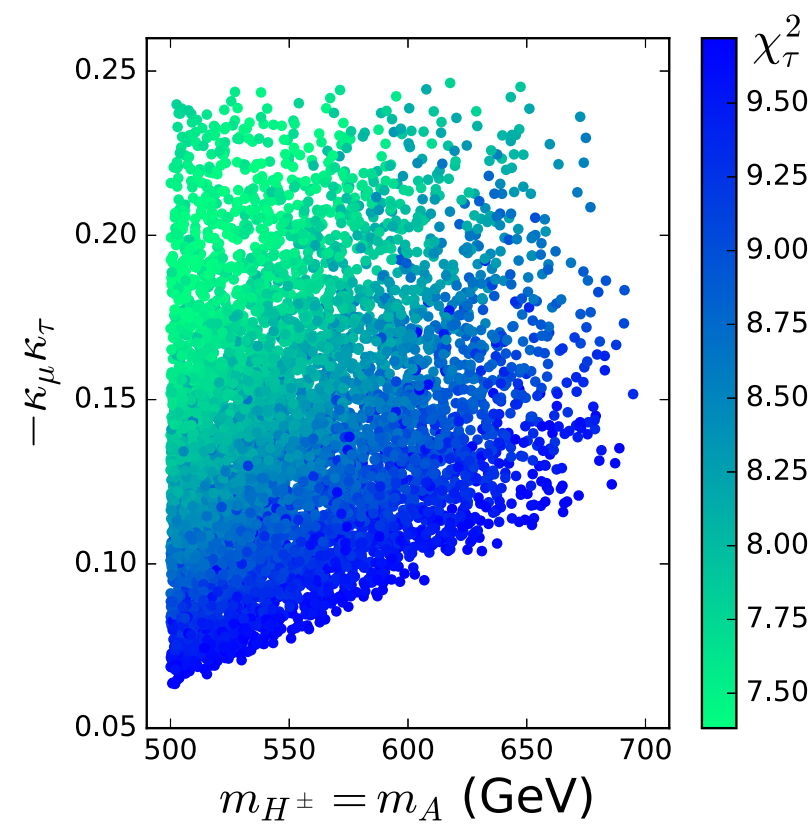

FIG. 2. The surviving samples fit the data of LFU in $\tau$ decay within the $2 \sigma$ range. All the samples satisfy the constraints of the theory and oblique parameters.

degree of freedom is removed in our calculation. In our discussions we require $\chi_{\tau}^{2}<9.72$, which corresponds to be within the $2 \sigma$ range for four observables, and is smaller than the $\mathrm{SM}$ value, $\chi_{\tau}^{2}(\mathrm{SM})=12.25$.

The measured values of the ratios of the leptonic $Z$ decay branching fractions are given as [29]

$$
\begin{aligned}
& \frac{\Gamma_{Z \rightarrow \mu^{+} \mu^{-}}}{\Gamma_{Z \rightarrow e^{+} e^{-}}}=1.0009 \pm 0.0028, \\
& \frac{\Gamma_{Z \rightarrow \tau^{+} \tau^{-}}}{\Gamma_{Z \rightarrow e^{+} e^{-}}}=1.0019 \pm 0.0032,
\end{aligned}
$$

with a correlation of +0.63 . In the model, the width of $Z \rightarrow \tau^{+} \tau^{-}$can have sizable deviation from the SM value due to the loop contributions of the extra Higgs bosons, because they strongly interact with charged leptons. The calculations of quantities in Eq. (20) are similar to Ref. [30].

After imposing the constraints of the theory and the oblique parameters, in Fig. 1 we show the surviving samples which are consistent with $\Delta a_{\mu}$ and $\Delta a_{e}$ at the $2 \sigma$ level. Both one-loop and two-loop diagrams give positive contributions to $\Delta a_{\mu}$. For $\Delta a_{e}$, the contributions of one-loop are positive and those of two-loop are negative. Only the contributions of two-loop can make $\Delta a_{e}$ to be within the $2 \sigma$ range. $\Delta a_{\mu}$ and $\Delta a_{e}$ respectively favor negative $\kappa_{\mu}$ and positive $\kappa_{e}$ for increasing $m_{H}$, and $m_{H}$ is required to be smaller than $320 \mathrm{GeV}$ from $\Delta a_{e}$. A large mass splitting between $m_{A}$ and $m_{H}$ can lead to sizable corrections to $\Delta a_{\mu}$ and $\Delta a_{e}$. Therefore, the right panel of Fig. 1 shows that $m_{A}$ is favored for increasing $m_{H}$, especially for a large $m_{H}$.

After imposing the constraints of the theory and the oblique parameters, we show the surviving samples with $\chi_{\tau}^{2}<9.72$ in Fig. 2. Such samples fit the data of LFU in $\tau$ decay within the $2 \sigma$ range. Because $\kappa_{\mu}$ is opposite in sign from $\kappa_{\tau}$, the second term of $\delta_{\text {tree }}$ in Eq. (17) is positive, which gives a well fit to $g_{\tau} / g_{e}$. Figure 2 shows that $\chi_{\tau}^{2}$ can be as low as 7.4, which is much smaller than the SM value (12.25). The value of $\chi_{\tau}^{2}$ decreases with an increase of $-\kappa_{\mu} \kappa_{\tau}$ and increases with $m_{H^{ \pm}}$.

In Fig. 3 we show the surviving samples after imposing the constraints of theory, the oblique parameters, $\Delta a_{\mu}, \Delta a_{e}$, the data of LFU in $\tau$ decay and $Z$ decay, and the direct searches at LHC. The model can give sizable corrections to $Z \rightarrow \tau^{+} \tau^{-}$for large $\kappa_{\tau}$ and mass splitting between $m_{A}$ and $m_{H}$. Therefore, the region of the small $m_{H}$ and large $\kappa_{\tau}$ is excluded by the data of LFU in $Z$ decay, as shown in the middle panel of Fig. 3. The left panel of Fig. 3 shows that the exclusion limits from the direct searches at LHC favor large $m_{H}, m_{A}$, and $m_{H^{ \pm}}$. After imposing the theoretical constraint and relevant experimental constraints, the model can explain the anomalies of $\Delta a_{\mu}, \Delta a_{e}$ and LFU in the $\tau$
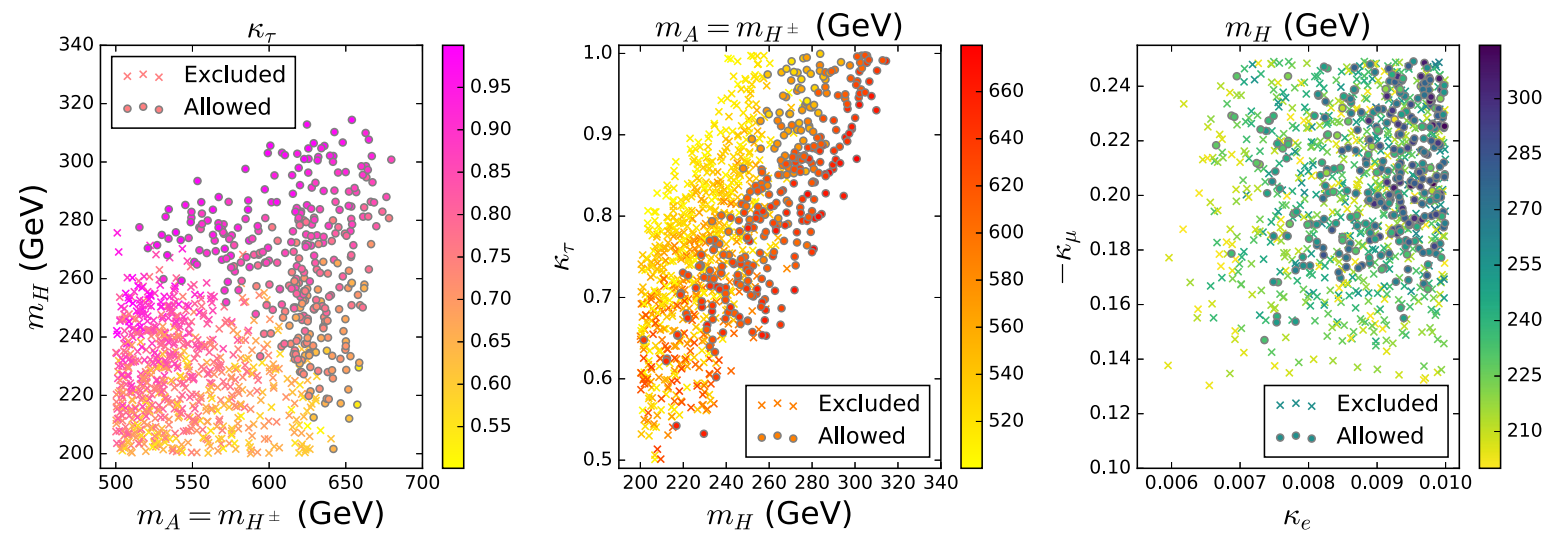

FIG. 3. The allowed samples (dots with gray edge) and excluded samples (crosses) by the direct search limits from the LHC at 95\% confidence level. The colors indicate $\kappa_{\tau}, m_{A}$ and $m_{H}$ in left, middle, and right panels, respectively. All the samples satisfy the constraints of theory, the oblique parameters, $\Delta a_{\mu}, \Delta a_{e}$, the data of LFU in $\tau$ decays, and $Z$ decay. 
decay in many parameter spaces of $200 \mathrm{GeV}<m_{H}<$ $320 \mathrm{GeV}, 500 \mathrm{GeV}<m_{A}=m_{H^{ \pm}}<680 \mathrm{GeV}, 0.0066<\kappa_{e}<$ $0.01,-0.25<\kappa_{\mu}<-0.147$, and $0.53<\kappa_{\tau}<1.0$. By normalizing event yields in the signal regions of Ref. [23] to higher luminosities, we find that these parameter spaces can be fully detected at $95 \%$ confidence level with about $80 \mathrm{fb}^{-1} 13 \mathrm{TeV}$ LHC data.

Note the $Z_{2}$ breaking term $\mu\left(\Phi_{1}^{\dagger} \Phi_{2}+\right.$ H.c. $)$ is inevitable when we consider the renormalization of one-loop divergent integral. Although it can be set to be zero at some energy scale, radiative corrections will regenerate it at different scales. We can denote it as the $\mu$ problem in our model. The vanishing of the $\mu$ term does not induce an enhanced symmetry so that nothing prevents it to be large via quantum corrections. However, we have to figure out that the two-Higgs-doublet model is not UV consistent theory since the Higgs mass hierarchy problem is not solved. These two hierarchy problems, i.e., $\mu$ problem and Higgs mass problem, motivate us to consider new physics around $\mathrm{TeV}$ such as supersymmetry, which will be studied in the future paper. Therefore, the intrinsic cutoff for quantum correction is around $\mathrm{TeV}$. The mixing mass term like " $h H$ " can be generated at one-loop by the exchange of SM leptons in the loop, but is sizably suppressed by the loop factor of $\frac{1}{16 \pi^{2}}$ and $h \tau \bar{\tau}$ coupling of $\frac{m_{\tau}}{v}$. For the cutoff of $\mathrm{TeV}$, we can obtain a small value of $\mu$ through the cancellation between the bare term and the quadratic loop correction. The price we paid is that we have to accept finetuning. As a result, we can still obtain a $Z_{2}$ symmetric model with $Z_{2}$ breaking terms being very small.

\section{CONCLUSION}

We have proposed a lepton-specific inert 2HDM, where an inert Higgs doublet field with a discrete $Z_{2}$ symmetry is introduced to the SM. Considering all the current theoretical and experimental constraints, we showed that our model can provide a simple explanation for the anomalies of muon $g-2$, electron $g-2$, and LFU of the $\tau$ decays in many viable parameter spaces.

\section{ACKNOWLEDGMENTS}

We thank Bin Zhu for helpful discussions. This work was supported by Projects No. 11475238, No. 11575152, No. 11647601, and No. 11875062 supported by the National Natural Science Foundation of China, by the Natural Science Foundation of Shandong province (ZR2017MA004, ZR2017JL002), by the Key Research Program of Frontier Science, CAS, and by the ARC Centre of Excellence for Particle Physics at the Tera-scale under Grant No. CE110001004.
[1] J. S. Schwinger, Phys. Rev. 73, 416 (1948).

[2] H. N. Brown et al. (Muon $g-2$ Collaboration), Phys. Rev. Lett. 86, 2227 (2001).

[3] T. Blum, P. A. Boyle, V. Gülpers, T. Izubuchi, L. Jin, C. Jung, A. Jüttner, C. Lehner, A. Portelli, and J. T. Tsang (RBC and UKQCD Collaborations), Phys. Rev. Lett. 121, 022003 (2018).

[4] R. H. Parker, C. Yu, W. Zhong, B. Estey, and H. Mueller, Science 360, 191 (2018).

[5] T. Aoyama, T. Kinoshita, and M. Nio, Phys. Rev. D 97, 036001 (2018).

[6] D. Hanneke, S. Fogwell, and G. Gabrielse, Phys. Rev. Lett. 100, 120801 (2008).

[7] D. Hanneke, S. F. Hoogerheide, and G. Gabrielse, Phys. Rev. A 83, 052122 (2011).

[8] Y. Amhis et al. (Heavy Flavor Averaging Group (HFAG) Collaboration), arXiv:1412.7515.

[9] T. Abe, R. Sato, and K. Yagyu, J. High Energy Phys. 07 (2015) 064.

[10] A. Crivellin, J. Heeck, and P. Stoffer, Phys. Rev. Lett. 116, 081801 (2016).

[11] E. J. Chun and J. Kim, J. High Energy Phys. 07 (2016) 110.

[12] G. F. Giudice, P. Paradisi, and M. Passera, J. High Energy Phys. 11 (2012) 113.
[13] H. Davoudiasl and W. J. Marciano, Phys. Rev. D 98, 075011 (2018).

[14] J.-J. Zhang, M. He, X.-G. He, and G. Li, arXiv:1807.11363.

[15] A. Crivellin, M. Hoferichter, and P. Schmidt-Wellenburg, Phys. Rev. D 98, 113002 (2018).

[16] J. Liu, C. E. M. Wagner, and X.-P. Wang, J. High Energy Phys. 03 (2019) 008.

[17] B. Dutta and Y. Mimura, Phys. Lett. B 790, 563 (2019).

[18] D. Eriksson, J. Rathsman, and O. Stål, Comput. Phys. Commun. 181, 189 (2010).

[19] J. Alwall, R. Frederix, S. Frixione, V. Hirschi, F. Maltoni, O. Mattelaer, H.-S. Shao, T. Stelzer, P. Torrielli, and M. Zaro, J. High Energy Phys. 07 (2014) 079.

[20] P. Torrielli and S. Frixione, J. High Energy Phys. 04 (2010) 110.

[21] D. Dercks, N. Desai, J. S. Kim, K. Rolbiecki, J. Tattersall, and T. Weber, Comput. Phys. Commun. 221, 383 (2017).

[22] A. M. Sirunyan et al. (CMS Collaboration), J. High Energy Phys. 03 (2018) 160.

[23] A. M. Sirunyan et al. (CMS Collaboration), J. High Energy Phys. 03 (2018) 166.

[24] A. M. Sirunyan et al. (CMS Collaboration), J. High Energy Phys. 03 (2018) 076.

[25] A. M. Sirunyan et al. (CMS Collaboration), J. High Energy Phys. 11 (2017) 029. 
[26] A. Dedes and H. E. Haber, J. High Energy Phys. 05 (2001) 006.

[27] D. Chang, W.-F. Chang, C.-H. Chou, and W.-Y. Keung, Phys. Rev. D 63, 091301 (2001).

[28] K. M. Cheung, C. H. Chou, and O. C. W. Kong, Phys. Rev. D 64, 111301 (2001).
[29] S. Schael et al. (ALEPH, DELPHI, L3, OPAL, SLD, LEP Electroweak Working Group, SLD Electroweak Group, SLD Heavy Flavour Group Collaborations), Phys. Rept. 427, 257 (2006).

[30] L. Wang, J. M. Yang, M. Zhang, and Y. Zhang, Phys. Lett. B 788, 519 (2019). 